\title{
Oral Session 9: Adaptation and Rehabilitation
}

\section{9-1 [\#3023]}

\section{Training to facilitate adaptation to novel sensory environments}

J.J. Bloomberg a, B.T. Peters ${ }^{b}$, A.P. Mulavara ${ }^{c}$, R.A. Brady $^{\text {b }}$, C.D. Batson ${ }^{\text {d }}$, R.J. Ploutz-Snyder ${ }^{c}$ and H.S. Cohen $^{\mathrm{e}}$

${ }^{a}$ Neuroscience Laboratories, NASA-Johnson Space Center, Houston, TX, USA

${ }^{\mathrm{b}}$ Wyle Integrated Science and Engineering Group, Houston, TX, USA

${ }^{\mathrm{c}}$ Universities Space Research Association Division of Space Life Sciences, Houston, TX, USA

${ }^{\mathrm{d}}$ MEI Technologies, Inc., Houston, TX, USA

${ }^{\mathrm{e}}$ Bobby R. Alford Department of Otolaryngology Head and Neck Surgery, Baylor College of Medicine, Houston, TX, USA

After spaceflight, the process of readapting to Earth's gravity causes locomotor dysfunction. We are developing a gait training countermeasure to facilitate adaptive responses in locomotor function. Our training system is comprised of a treadmill placed on a motion-base facing a virtual visual scene that provides an unstable walking surface combined with incongruent visual flow designed to train subjects to rapidly adapt their gait patterns to changes in the sensory environment. The goal of our present study was to determine if training improved both the locomotor and dual-tasking ability responses to a novel sensory environment and to quantify the retention of training. Subjects completed three, 30-minute training sessions during which they walked on the treadmill while receiving discordant support surface and visual input. Control subjects walked on the treadmill without any support surface or visual alterations. To determine the efficacy of training all subjects were then tested using a novel visual flow and support surface movement, not previously experienced during training. This test was performed 20 minutes, 1 week, 1, 3 and 6 months after the final training ses- sion. Stride frequency and auditory reaction time were collected as measures of postural stability and cognitive effort, respectively. Subjects who received training showed less alteration in stride frequency and auditory reaction time compared to controls. Trained subjects maintained their level of performance over six months. We conclude that, with training, individuals become more proficient at walking in novel discordant sensorimotor conditions and are able to devote more attention to competing tasks.

Acknowledgement: This work was supported by the National Space Biomedical Research Institute through NASA NCC 9-58.

\section{9-2 [\#3022]}

Different profiles of vestibular habituation in pilots and common people

L.-J. Wang, C. Tan, Y. Cao, Q Zhao, G.-H. Tang, S.Y. He and D.-B. Niu

State Keylab of Space Fundamental and Application Medicine, China Astronaut Research and Training Center, Beijing, P.O. Box 5132(15), 100094, Beijing, China.E-mail: wlj823@sina.com

Objective: The different profiles of vestibular habituation in pilots and common people were explored after their Coriolis test selection.

Methods: 12 healthy men and 14 pilot students passed the Coriolis test selection and participated in this experiment. They were exposed to five-day continuous or intermittent Coriolis adaptation training. The vestibular autonomic responses such as motion sickness symptom, ECG, EGG, EOG, facial skin infrared images and blood biochemistry indexes elicited by Coriolis test were monitored at 1st day, 1st week, 2nd week, 4th week, 5th week, 9th week and 14th week after training.

Results: It was found that subjects all passed the $10 \mathrm{~min}$ continuous or $15 \mathrm{~min}$ intermittent Coriolis adap- 
tation training. For common healthy men, after Coriolis training the low motion sickness symptom scores can maintain 1 or 2 weeks, the habituation of autonomic responses was about 1 week, the endocrine system response held for 2 weeks, the habituation of facial skin was about 2 weeks and the slow phase speed of EOG can maintain for 3 months. For pilot students, the low motion sickness symptom scores lasted for 5 weeks, the EGG habituation was about 2 weeks, the slow phase speed of EOG can maintain for 9 weeks, but the autonomic responses had no habituation effects.

Conclusion: It was suggested that pilots were a special group of people, and the profile of vestibular habituation presented a dynamic changes expression in respective aspects of vestibular autonomic responses.

Acknowledgement: This work was supported by Advanced Space Medico-Engineering Research Project of China (No. 2007SY5406002) and the National Science Foundation of China (Grant No. 31070914).

\section{References}

[1] G. Clement et al., Eur J Appl Physiol 85 (2001), 539-545.

[2] G. Clement et al., Exp Brain Res 190 (2008), 307-315.

[3] W. Liu et al., Chinese Space Aviat Med 16 (2005), 188-190.

[4] G. Clement et al., J Vestib Res 17 (2007), 237-247.

\section{9-3 [\#3052]}

Enhancing vestibular function by imperceptible electrical stimulation

J.M. Serrador ${ }^{\mathrm{a}}$, M.C. Geraghty ${ }^{\mathrm{b}}$, B.M. Deegan ${ }^{\mathrm{c}}$ and S.J. Wood ${ }^{\mathrm{d}}$

${ }^{a}$ War Related Illness and Injury Study Center, NJ VA Health Care System, East Orange, NJ and Harvard Medical, Boston, MA, USA

${ }^{\mathrm{b}}$ University College Dublin, Dublin, Ireland

${ }^{\mathrm{c}}$ National University of Ireland Galway, Galway, Ireland

${ }^{\mathrm{d}}$ NASA Johnson Space Centre and Universities Space Research Association, Houston, TX, USA

Loss of vestibular function occurs with both disease and aging, but currently there are limited options to treat this loss. Stochastic resonance (SR) is a counterintuitive phenomenon in which the addition of a suitable noise level can enhance the detection of weak signals. SR has been shown to improve sensory function in a number of systems. Since loss of vestibular function is likely due to loss of hair cells, and thus a reduced sensory input, we hypothesized that SR noise would enhance this response and thus improve vestibular function in elderly subjects. To test this we applied SR noise at sub threshold levels to electrodes located over the mastoid process during sinusoidal roll tilt of \pm 25 degrees in 8 elderly subjects and 7 younger subjects. We examined ocular counter-roll (OCR) during tilt, a reflexive eye rotation in the opposite direction mediated through the vestibular system. We found that all elderly subjects increased OCR (Mean 23\%, Range 4-60\%) in contrast to younger subjects (Mean $+1 \%$, Range $-16-27 \%$ ).

The increase was linearly related to baseline OCR ( $R=0.67, P=0.007$ ), indicating that subjects with impaired vestibular function showed the greatest benefit, regardless of age. These findings demonstrate that galvanic stimulation with SR noise is able to restore vestibular function in those with impaired function. Since stimulation was sub threshold, these data suggest that application of SR galvanic stimulation may provide a new treatment alternative to restore vestibular function.

Acknowledgement: This work was supported by NASA grant NNJ04HI13G (Serrador).

\section{9-4 [\#3015]}

\section{Vestibular-somatosensory convergence in head movement control during locomotion after long-duration space flight}

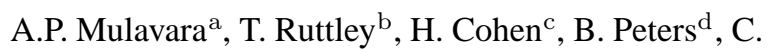
Miller $^{\mathrm{d}}$, R. Brady ${ }^{\mathrm{d}}$, L. Merkle ${ }^{\mathrm{d}}$ and J.J. Bloomberg ${ }^{\mathrm{b}}$

${ }^{\mathrm{a}}$ Universities Space Research Association, Houston, TX, USA

${ }^{\mathrm{b}}$ NASA Johnson Space Center, Houston, TX, USA

${ }^{\mathrm{c}}$ Baylor College of Medicine, Houston, TX, USA

${ }^{\mathrm{d}}$ Wyle Integrated Science and Engineering Group, Houston, TX, USA

Exposure to the microgravity conditions of space flight induces adaptive modification in the control of vestibular-mediated reflexive head movement during locomotion after space flight. Space flight causes astronauts to be exposed to adaptation in both the vestibular and body load- sensing (BLS) somatosensory systems. The goal of these studies was to examine the contributions of vestibular and BLS somatosensory influences on head movement control during locomotion after long-duration space flight. Subjects were asked to 
walk on a treadmill driven at $1.8 \mathrm{~m} / \mathrm{s}$ while performing a visual acuity task. Data were collected using the same testing protocol from three independent subject groups; 1) normal subjects before and after exposure to $30 \mathrm{~min}$ utes of $40 \%$ bodyweight unloaded treadmill walking, 2) bilateral labyrinthine deficient (LD) patients and 3) astronauts who performed the protocol before and after long duration space flight. Motion data from head and trunk segmental motion data were obtained to calculate the angular head pitch (HP) movements during walking trials while subjects performed the visual task, to estimate the contributions of vestibular reflexive mechanisms in HP movements. Results showed that exposure to unloaded locomotion caused a significant increase in HP movements, whereas in the LD patients the HP movements were significantly decreased. Astronaut subjects' results showed a heterogeneous response of both increases and decreases in the amplitude of HP movement. We infer that BLS-mediated somatosensory input centrally modulates vestibular input and can adaptively modify head-movement control during locomotion. Thus, space flight may cause a central adaptation of the converging vestibular and body load-sensing somatosensory systems.

\section{9-5 [\#3032]}

\section{Comparison of postural recovery after short and long duration spaceflights}

S.J. Wood ${ }^{\mathrm{a}, \mathrm{b}}$, J. Fiedler ${ }^{\mathrm{a}, \mathrm{b}}$, L.C. Taylor ${ }^{\mathrm{a}, \mathrm{c}}$, D. Sayen$\mathrm{ko}^{\mathrm{d}}$, I. Kozlovskaya ${ }^{\mathrm{d}}$, F.O. Black ${ }^{\mathrm{e}}$ and W.H. Paloski ${ }^{\mathrm{a}, \mathrm{f}}$ ${ }^{a}$ NASA Johnson Space Center, Houston, TX, USA

${ }^{\mathrm{b}}$ Universities Space Research Association, Houston, TX, USA

${ }^{\mathrm{c}}$ Wyle Integrated Science and Engineering Group, Houston, TX, USA

${ }^{\mathrm{d}}$ Institute of Biomedical Problems, Moscow, Russia e Legacy Health System, Portland, OR, USA

${ }^{\mathrm{f}}$ University of Houston, Houston, TX, USA

Introduction: Post-flight postural ataxia reflects adaptive changes to vestibulo-spinal reflexes and control strategies adopted for movement in weightlessness. Quantitative measures obtained during computerized dynamic posturography (CDP) from US and Russian programs provide insight into the effect of spaceflight duration in terms of both the initial decrements and recovery of postural stability.

Methods: CDP data were obtained on 117 crewmembers after Shuttle flights lasting 4-17 days, and on 64 crewmembers following long-duration missions lasting 48-380 days. Although the number and timing of sessions varied, the goal was to characterize postural recovery by pooling similar measures from different research and flight medicine programs. This report focuses on eyes closed, head erect conditions with either a fixed or sway-referenced base of support. A smaller subset of subjects repeated the sway- referenced condition while making pitch head movements $\left( \pm 20^{\circ}\right.$ at $0.33 \mathrm{~Hz}$ ). Equilibrium scores were derived from peakto-peak anterior-posterior sway. Fall probability and recovery curves were modeled using Bayesian statistical methods.

Results: The standard Romberg condition was the least sensitive. Longer duration flights led to larger decrements in stability with sway-reference support during the first 1-2 days, although the time course of recovery was similar across flight duration with head erect. Head movements led to increased incidence of falls during the first week, with a significantly longer recovery after long duration flights.

Conclusions: The diagnostic assessment of postural instability, and differences in the time course of postural recovery between short and long flight durations, are more pronounced during unstable support conditions requiring active head movements. 